\title{
Immunogenomic determinants of tumor microenvironment correlate with superior survival in high- risk neuroblastoma
}

To cite: Bao R, Spranger S, Hernandez K, et al. Immunogenomic determinants of tumor microenvironment correlate with superior survival in high-risk neuroblastoma. Journal for ImmunoTherapy of Cancer 2021:9:e002417. doi:10.1136/jitc-2021-002417

- Additional supplemental material is published online only. To view, please visit the journal online (http://dx.doi.org/10. 1136/jitc-2021-002417).

Accepted 17 May 2021

\section{Check for updates}

(c) Author(s) (or their employer(s)) 2021. Re-use permitted under CC BY-NC. No commercial re-use. See rights and permissions. Published by BMJ.

For numbered affiliations see end of article.

\section{Correspondence to} Dr Ami V Desai; adesai12@peds.bsd.uchicago. edu

Riyue Bao (1) , ,2 Stefani Spranger, ${ }^{3,4}$ Kyle Hernandez, ${ }^{5,6}$ Yuanyuan Zha, ${ }^{6}$ Peter Pytel, ${ }^{7}$ Jason J Luke (1) , ,,2 Thomas F Gajewski, ${ }^{6,7}$ Samuel L Volchenboum, ${ }^{8}$ Susan L Cohn, ${ }^{8}$ Ami V Desai ${ }^{8}$

\section{ABSTRACT}

Background Tumor-infiltrating $\mathrm{CD} 8^{+} \mathrm{T}$ cells and neoantigens are predictors of a favorable prognosis and response to immunotherapy with checkpoint inhibitors in many types of adult cancer, but little is known about their role in pediatric malignancies. Here, we analyzed the prognostic strength of T cell-inflamed gene expression and neoantigen load in high-risk neuroblastoma. We also compared transcriptional programs in T cell-inflamed and non-T cell-inflamed high-risk neuroblastomas to investigate possible mechanisms of immune exclusion.

Methods A defined T cell-inflamed gene expression signature was used to categorize high-risk neuroblastomas in the Therapeutically Applicable Research to Generate Effective Treatments (TARGET) program $(n=123)$, and the Gabriella Miller Kids First (GMKF) program ( $n=48)$ into T cell-inflamed, non- $T$ cellinflamed, and intermediate groups. Associations between the T cell-inflamed and non-T cell-inflamed group, MYCN amplification, and survival were analyzed by Cox proportional hazards models. Additional survival analysis was conducted after integrating neoantigen load predicted from somatic mutations. Pathways activated in non-T cellinflamed relative to $\mathrm{T}$ cell-inflamed tumors were analyzed using causal network analysis.

Results Patients with T cell-inflamed high-risk tumors showed improved overall survival compared with those with non- $\mathrm{T}$ cell-inflamed tumors $(\mathrm{p}<0.05)$, independent of MYCN amplification status, in both TARGET and GMKF cohorts. Higher neoantigen load was also associated with better event-free and overall survival $(p<0.005)$ and was independent of the T cell-inflamed signature. Activation of MYCN, ASCL1, SOX11, and KMT2A transcriptional programs was inversely correlated with the T cell-inflamed signature in both cohorts.

Conclusions Our results indicate that tumors from children with high-risk neuroblastoma harboring a strong T cell-inflamed signature have a more favorable clinical outcome, and neoantigen load is a prognosis predictor, independent of T cell inflammation. Strategies to target SOX11 and other signaling pathways associated with non-T cell-inflamed tumors should be pursued as potential immune-potentiating interventions.

\section{BACKGROUND}

The presence of effector $\mathrm{T}$ cells in the tumor microenvironment has been associated with improved survival in adults with many types of cancer. ${ }^{1-3}$ Several studies of melanoma and other solid tumors have demonstrated that expression of dendritic cell (DC) and CD8 ${ }^{+}$ $\mathrm{T}$ cell-associated genes, or a $\mathrm{T}$ cell-inflamed gene signature, is correlated with favorable prognosis and response to immunotherapy with checkpoint blockade therapy or tumor vaccines. ${ }^{4-8} \mathrm{~T}$ cell-inflamed tumors are characterized by type I interferon (IFN) activation, immune potentiating chemokines, antigen presentation, cytotoxic effector molecules, and activated $\mathrm{CD}^{+} \mathrm{T}$ cells. ${ }^{9}$ The inflamed tumor microenvironment is additionally characterized by IFN-induced inhibitory pathways such as programmed death-ligand 1 (PDL1) and indoleamine-2, 3 dioxygenase, and higher proportions of $\mathrm{FOXP}^{+}$regulatory $\mathrm{T}$ cells. ${ }^{9}$ Other known predictors of response to immunotherapy include, but are not limited to, a high tumor mutational burden $(\mathrm{TMB})^{10}$ and a high neoantigen load. ${ }^{11}$ While TMB and neoantigen load often highly correlate with each other, ${ }^{12}$ previous studies have demonstrated both markers have low correlation with the presence of $\mathrm{T}$ cell inflammation, ${ }^{101213}$ and TMB (or neoantigen load) and $\mathrm{T}$ cell-inflamed gene expression may represent non-redundant predictive biomarkers of immune checkpoint inhibitors efficacy. ${ }^{14}$

In contrast, resistance to immunotherapy has been correlated with tumors that lack the $\mathrm{T}$ cell-inflamed signature. There is increasing evidence that signaling pathways intrinsic to the neoplastic cells may impair the local immune response in tumors. Tumor cellintrinsic activation of the $\mathrm{WNT} / \beta$-catenin pathway has been associated with a lack of $\mathrm{T}$ 
cell infiltration in melanoma, bladder cancer, and more broadly across cancer. ${ }^{15-17}$ Activation of the phosphoinositide 3-kinase (PI3K) signaling pathway through loss-offunction mutations in phosphatase and tensin homolog (PTEN) can likewise mediate a non-T cell-inflamed tumor microenvironment in melanoma, ${ }^{18}$ and inactivation of LKB1 can have a similar effect in lung adenocarcinoma. ${ }^{19}$ Further, in lymphoma, diminished activation and recruitment of $\mathrm{T}$ cells have been reported with MYC activation, largely through inhibition of macrophage activation. ${ }^{20}$ MYC and several other activated transcriptional pathways have more broadly been associated with non-T cell-inflamed tumors across cancer types. ${ }^{21}$

In contrast to adult cancers, pediatric neoplasms have low mutational burden and most are non-T cell-inflamed, with scarce tumor-infiltrating lymphocytes (TILs) among anti-inflammatory M2 tumor-associated macrophages (TAMs). ${ }^{22}{ }^{23}$ Although the response to immune checkpoint inhibition is poor for many pediatric cancers, ${ }^{24} 25$ post-consolidation immunotherapy with monoclonal antibodies targeting the GD2 ganglioside combined with cytokines significantly improves survival for children with high-risk neuroblastoma. ${ }^{26}$ Further, high response rates were also reported in newly diagnosed patients in a single institutional study with induction chemotherapy combined with anti-GD2 antibody, ${ }^{27}$ and significant antitumor immunity was observed in a Children's Oncology Group (COG) clinical trial testing irinotecan and temozolomide combined with anti-GD2 antibody and GM-CSF in patients with relapsed/refractory neuroblastoma. ${ }^{28} 29$

The immunobiology of the neuroblastoma microenvironment is an emerging field. To increase our understanding about how immunogenomic determinants influence neuroblastoma phenotype, we analyzed the correlation between patient survival and T cell-inflamed gene expression and neoantigen load in tumor. We demonstrate that both biomarkers are prognostic in children with high-risk neuroblastoma and identify tumor-intrinsic oncogenic signaling pathways activated in neuroblastomas with a non-T cell-inflamed phenotype. These findings enhance a framework, whereby $\mathrm{T}$ cellinflamed expression and neoantigen load can provide new prognostic information to inform treatment decisions, and may also lead to the development of future immune therapeutic interventions.

\section{METHODS}

\section{Study cohorts and datasets}

Two neuroblastoma cohorts were analyzed. The discovery cohort included patients from Therapeutically Applicable Research to Generate Effective Treatments (TARGET) program ( $\mathrm{n}=149 ; 123$ high-risk) (dbGAP accession ID phs000218.v22.p8) (online supplemental table 1). RNAseq paired-end (PE) FastQ files, whole exome sequencing (WES) alignment BAM files, somatic mutation MAF (Mutation Annotation Format) files, and clinical data were downloaded from Genomic Data Commons $(\text { GDC })^{30} \quad$ (https://portal.gdc.cancer.gov) (accessed 07/2017). The validation cohort included patients with clinical information in the International Neuroblastoma Risk Group (INRG) Data Commons ${ }^{31}$ and tumor RNAseq data in the Gabriella Miller Kids First (GMKF) program ( $\mathrm{n}=198 ; 48$ high-risk) (online supplemental table 2). Universal system identification (USI) numbers were used to link the datasets. Access to RNAseq PE FastQ files in GMKF could not be obtained at the time of study, and therefore, preprocessed gene expression TSV files from the GMKF data portal (https://kidsfirstdrc.org/) (accessed 08/2020) were used for analysis. Of the 209 patients identified, 11 were determined by USI number to also be included in the discovery cohort, hence were excluded from the validation cohort; 198 were kept for validation (online supplemental table 2 ).

\section{RNAseq gene expression quantification}

The quality of raw sequencing reads was assessed by FastQC $^{32}$ (V.0.11.5) for the tumor samples in the discovery cohort. Read counts were quantified at transcript level using Kallisto ${ }^{33}$ (V.0.44.0) with human reference assembly GRCh38 and Gencode gene annotation (V.28), summarized into gene level using tximport ${ }^{34}$ (V.1.4.0), normalized by trimmed mean of M-values (TMM) method, and $\log _{2}$-transformed.

\section{Identification of T cell-inflamed and non-T cell-inflamed tumor groups}

Using a defined $\mathrm{T}$ cell-inflamed gene expression signature, ${ }^{13} 21$ the tumors in the discovery cohort were categorized into three groups ( $\mathrm{T}$ cell-inflamed, non- $\mathrm{T}$ cell-inflamed, and intermediate) using consensus clustering methods following previous protocols. ${ }^{13}$ In brief, an expression matrix consisting of the 160 genes from the T cell-inflamed signature ${ }^{21}$ was subset from the TMMnormalized and $\log _{2}$-transformed RNAseq gene expression quantification matrix and was used to cluster tumors into 12 clusters by ConsensusClusterPlus (V.1.42.0) using hierarchical clustering with Euclidean distance and Ward.D2 linkage (2000 bootstraps and $80 \%$ usage of gene features). Tumors were then assigned with each of the three immune groups based on high, low, or intermediate expression of the T cell-inflamed signature. The number of clusters was determined using the elbow method.

\section{Mapping of T cell-inflamed and non-T cell-inflamed tumor groups between discovery and validation cohorts}

The assignment of tumor groups in the discovery cohort cannot be migrated directly to that of tumors in the validation cohort due to the relative nature of gene expression data without spike-in controls. To address this issue, we projected $\mathrm{T}$ cell-inflamed gene expression of the validation cohort to the space of the discovery cohort using 11 patients that overlap between the two cohorts. First, we normalized and $\log _{2}$-transformed gene expression within each cohort. We calculated a T cell-inflamed score for each tumor, defined as the mean expression of all 
genes from the signature. ${ }^{21}$ Then, we fit a linear regression model on the T cell-inflamed scores of the discovery cohort and validation cohort using tumors from the 11 overlapping patients, Score $_{T}=-0.7798+1.2418 \times$ Score $_{G}$ (adjusted $\mathrm{R}^{2}=0.967$ ), where Score $_{T}$ represents $\mathrm{T}$ cellinflamed score of the discovery cohort ( $\mathrm{T}$ as TARGET), and Score $_{G}$ represents T cell-inflamed score of the validation cohort ( $\mathrm{G}$ as GMKF). We used this model to convert all $\mathrm{T}$ cell-inflamed scores of tumors in the validation cohort $\left(\right.$ Score $\left._{G}\right)$ to values comparable to that of the discovery cohort $\left(\mathrm{Score}_{\mathrm{T}}\right)$, then sorted all tumors by T cellinflamed scores lower to higher. Lastly, we assigned new tumor groups to the validation cohort based on existing tumor groups from the discovery cohort, employing the rules as follows: for all tumors harboring a score less than or equal to that of the last non-T cell-inflamed tumor on the sorted list, assign as non-T cell-inflamed; for all tumors harboring a score greater than or equal to that of the first $\mathrm{T}$ cell-inflamed tumor on the sorted list, assign as $\mathrm{T}$ cell-inflamed; otherwise, assign as intermediate.

\section{Differential gene expression detection and pathway activation prediction}

For the discovery cohort analysis, we focused on 19,883 protein-coding genes defined in Gencode annotation (V.28) and followed the protocol established in our previous work. ${ }^{21}$ In brief, after removing genes with low expression (defined as CPM (counts per million of mapped reads) $\leq 3$ ), 15,580 genes with CPM>3 in at least 30 tumors were TMMnormalized and $\log _{2}$-transformed. Differentially expressed genes (DEGs) comparing non-T cell-inflamed with $\mathrm{T}$ cellinflamed groups were identified using Linear Models for Microarray Data (limma) voom ${ }^{35}$ method with precision weights (V.3.36.2) and filtered by false discovery rate (FDR)adjusted $\mathrm{p}<0.05$, and fold change $\geq 1.5$ or $\leq-1.5$. Upstream transcriptional regulators and change of direction (activation or inhibition) as a result of target molecules (encoded by DEGs) were predicted using Ingenuity Pathway Analysis (IPA) (QIAGEN, Germany) causal network analysis ${ }^{36}$ with the curated Ingenuity Knowledge Base (accessed 12/2017). Transcriptional programs activated in non-T cell-inflamed relative to $\mathrm{T}$ cell-inflamed tumors were filtered at overlap $\mathrm{p}<0.05$ (measuring the enrichment of target molecules in the dataset) and z-score $\geq 2.0$ (measuring the predicted activation level of the pathways). For the validation cohort analyses, preprocessed RNAseq expression data downloaded from the GMKF data portal was quantified using Kallisto $^{33}$ (V.0.44.0), and the per-tumor gene expression files were downloaded and aggregated into cohort level, TMMnormalized, and $\log _{2}$-transformed for further analysis.

\section{Somatic mutation detection, HLA genotyping, and neoantigen prediction}

For the discovery cohort, the somatic mutations were harmonized using four somatic variant callers (MuTect2, VarScan2, SomaticSniper, and MuSE).$^{30}$ After rigorous filtering following GDC's guidelines (https://docs.gdc. cancer.gov/Data/File_Formats/MAF_Format), somatic variants that were detected by at least two callers and passed all the filters were selected for further analysis. Total TMB was defined as the total number of nonsynonymous somatic mutations (NSSMs), those that were predicted to alter protein sequence in tumor (insertions/deletions, missense/nonsense/stopgain mutations, and those that modify splicing sites). Putative neoantigens were predicted from NSSMs using netMHCpan ${ }^{37}$ (V.4.0), filtered by gene expression from the RNAseq data described as follows. Patients' major histocompatibility complex (MHC) class I haplotypes were predicted from WES of germline DNA using Optitype (V.1.3.1). Nine-mer peptides were generated from the mutated site through a sliding window approach using in-house python scripts. Our previous work had suggested that peptides of SYFPEITHI ${ }^{38}$ mutant score $>25$ or delta score (mutant - wildtype) $>5$ bind to MHC class I molecules. ${ }^{13} \mathrm{In}$ this study, we used netMHCpan that covers more human leukocyte antigen (HLA) genotypes than SYFPEITHI. To select neoantigens that are likely to have strong binding affinity to HLA-A molecules and expressed in tumor, we filtered for 9-mer peptides of netMHCpan mutant score $>0.638$ (equivalent to IC $50 \mathrm{nmol}$, strong binding) or delta score $>0.070$ (correlated with SYFPEITHI delta score 5) and derived from genes upregulated compared with the median of its expression across all tumors.

\section{T cell-inflamed gene expression and pathway score calculation}

For each tumor, a T cell-inflamed score was computed as the mean expression of the 160 genes involved in the signature after scaling and centering across all tumor samples. ${ }^{21}$ A pathway activation score was calculated to each tumor following our published protocol, ${ }^{17} 21$ requiring at least $50 \%$ of the pathway-specific target molecules to be upregulated in a tumor sample (relative to its median expression across all tumor samples) in non- $\mathrm{T}$ cell-inflamed relative to T cell-inflamed group. For pathways in which less than 10 target molecules were present, 5 or more molecules were required to be upregulated to classify the pathway as activated. For pathways in which less than 5 target molecules were present, only pathways with all molecules upregulated were classified as activated. In addition, for each pathway identified in this study (MYCN, ASCL1, SOX11, and KMT2A), the expression level of a pathway was defined by the mean expression of all target molecules from this pathway, which was then used to correlate with the T cell-inflamed gene expression across all tumors by Spearman's correlation.

\section{Survival analysis}

Cox proportional hazards $(\mathrm{PH})$ models were used to test the association between the tumor group ( $\mathrm{T}$ cell-inflamed, non-T cell-inflamed, and intermediate) and the survival outcome (event-free survival (EFS); overall survival (OS)) in the discovery $(\mathrm{n}=118$ high-risk patients diagnosed between 2000 and 2010) and validation cohorts $(\mathrm{n}=17$ high-risk patients with survival data available) using $R$ 
package survival (function $\operatorname{coxph}$ (V.2.41.3). Univariable and multivariable Cox PH models were used to assess the significance of tumor group as a single predictor or after adjusting for covariates including age, MYCN status, and ploidy. In addition, Kaplan-Meier (KM) estimator with log-rank test was performed using $\mathrm{R}$ package survminer (V.0.4.2).

\section{Immunohistochemistry immunofluorescence staining}

Immunofluorescence (IF) staining on human neuroblastoma tumors was performed by the Human Immunologic Monitoring Core Facility at The University of Chicago using tissue from 17 intermediate or high-risk neuroblastomas (5 MYCN-amplified and 12 MYCN-non-amplified). Briefly, slides were baked, cleared, and rehydrated. After heat-inducted epitope retrieval, the slides were placed in a humidity chamber, blocked by $10 \%$ donkey serum for 1 hour, incubated with anti-CD8 Ab (Dako, M7103) at 1:100 dilution for 1 hour, followed by Cy3 donkey anti-Mouse IgG (Jackson Immunological Research Lab, 715-165-150) at 1:500 dilution for 1 hour. The slides then incubated with anti-Batf3 Ab (Novus, AF7437) at 1:40 dilution for 1 hour, followed by Cy5 donkey anti-Rabbit IgG (Jackson Immunological Research Lab, 711-175-152) at 1:200 dilution for 1 hour. After thorough wash, slides were incubated in DAPI and mounted with Fluoromount (Sigma, F4680). Images of the slides were taken using a Leica SP8 laser scanning confocal microscope at Integrated Light Microscopy Core Facility. A pathologist (PP) scored tumors for intensity and distribution of $\mathrm{CD} 8^{+}$cells and Batf3 ${ }^{+}$cells in a blinded fashion.

\section{Statistical analysis}

For analysis of contingency tables including comparison of tumor sample frequency between groups, Fisher's exact test was used. Differential gene expression analysis between groups were performed using empirical Bayes regression models in limma voom with precision weights. For multiple comparisons, p-value was adjusted using Benjamini-Hochberg FDR correction for multiple testing. ${ }^{39}$ Spearman's correlation $\rho$ was used for measuring statistical dependence between normalized and $\log _{2}$-transformed expression level of different genes and between gene expression of the $\mathrm{T}$ cell-inflamed signature and pathways. $\mathrm{p}<0.05$ was considered statistically significant. Statistical analysis was performed using $\mathrm{R}$ (V.3.5.2) and Bioconductor (release 3.8).

\section{RESULTS}

\section{A T cell-inflamed gene expression signature defines three distinct groups in neuroblastoma}

Using a defined T cell-inflamed gene expression signature, we categorized the 149 primary neuroblastoma tumors from the discovery cohort (TARGET) into three subsets (figure 1A). High expression of T cell signature genes ( $\mathrm{T}$ cell-inflamed) was detected in $57(38.3 \%)$ tumors, low or no expression (non-T cell-inflamed) was identified in 45
(30.2\%) tumors, and 47 (31.5\%) had intermediate levels of expression (intermediate) (table 1). In the validation cohort ( $\mathrm{n}=198, \mathrm{GMKF}), 89(44.9 \%)$ were categorized as T cell-inflamed; 55 (27.8\%) were non-T cell-inflamed; 54 $(27.3 \%)$ were intermediate (figure $1 \mathrm{~B}$, table 2$)$. In the discovery cohort, 123 of 149 patients were classified as high-risk, whereas 48 of 198 patients in validation cohort have high-risk neuroblastoma. ${ }^{40}$ In analyses restricted to high-risk patients, $53(43.1 \%)$ and $23(47.9 \%)$ were categorized as T cell-inflamed in the discovery and validation cohorts, respectively, and $33(26.8 \%)$ and $18(37.5 \%)$ were categorized as non-T cell-inflamed.

In the discovery cohort, MYCNamplification was significantly more prevalent in the non-T cell-inflamed tumors $(17 / 45,37.8 \%)$ compared with the $\mathrm{T}$ cell-inflamed tumors $(3 / 56,5.4 \%)(\mathrm{p}=0.000080$, odds ratio $[\mathrm{OR}]=10.5$, two-sided Fisher's exact test). Additionally, patients diagnosed at age $<18$ months had tumors that were enriched in the non-T cell-inflamed tumor group (12/45, $26.7 \%$ non-inflamed vs $6 / 57,10.5 \%$ inflamed; $p=0.040$, $\mathrm{OR}=3.06)$. The enrichment of $M Y C N$ amplification in non-T cell-inflamed tumors was also observed in the validation cohort $(14 / 54,25.9 \%$ non-inflamed vs $4 / 89,4.5 \%$ inflamed; $p=0.00038, O R=7.33)$. In addition, patients $<18$ months of age in the validation cohort had tumors that were enriched in the non-T cell-inflamed tumor group (40/54, 74.1\% non-inflamed vs 44/89, 49.4\% inflamed; $\mathrm{p}=0.0049, \mathrm{OR}=2.90$ ).

\section{T cell-inflamed gene expression is prognostic of survival in high-risk neuroblastoma}

We analyzed EFS and OS according to the level of expression of the $\mathrm{T}$ cell-inflamed signature in 118 high-risk patients diagnosed between 2000 and 2010 from the discovery cohort, which consists of $51 \mathrm{~T}$ cell-inflamed, 33 non-T cell-inflamed, and 34 intermediate tumors. In Cox PH univariable models, patients with T cell-inflamed tumors had significantly better OS compared with those with non- $\mathrm{T}$ cell-inflamed tumors $(\mathrm{p}=0.043$, hazard ratio $[\mathrm{HR}]=0.56$ ) (table 3 ). A similar trend was observed in EFS but the results did not reach statistical significance ( $\mathrm{p}=0.17, \mathrm{HR}=0.69) \quad$ (table 3). Similar to other high-risk cohorts, ${ }^{41} 42$ MYCN status was not statistically significantly associated with OS or EFS (OS: $\mathrm{p}=0.38$; EFS: $\mathrm{p}=0.58$ ) (table 3). However, OS, but not EFS, was better for highrisk patients with hyperdiploid neuroblastoma compared with those with diploid tumors (OS: $\mathrm{p}=0.05$; $\mathrm{EFS}$ : $\mathrm{p}=0.10$ ) (table 3). In Cox PH multivariable models adjusting for age, MYCN status, and ploidy, the T cell-inflamed signature maintained independent statistical significance for OS $(p=0.035, H R=0.48)$. Stage and histology were not included in the multivariable analysis because the highrisk patients had predominantly stage 4 disease and unfavorable histology tumors $(117 / 118,99.1 \%$ as stage 4; 107/110, 97.3\% with unfavorable histology tumors, 8 unknown).

In the discovery cohort, the $\mathrm{T}$ cell-inflamed and intermediate groups showed similar probabilities of survival. 

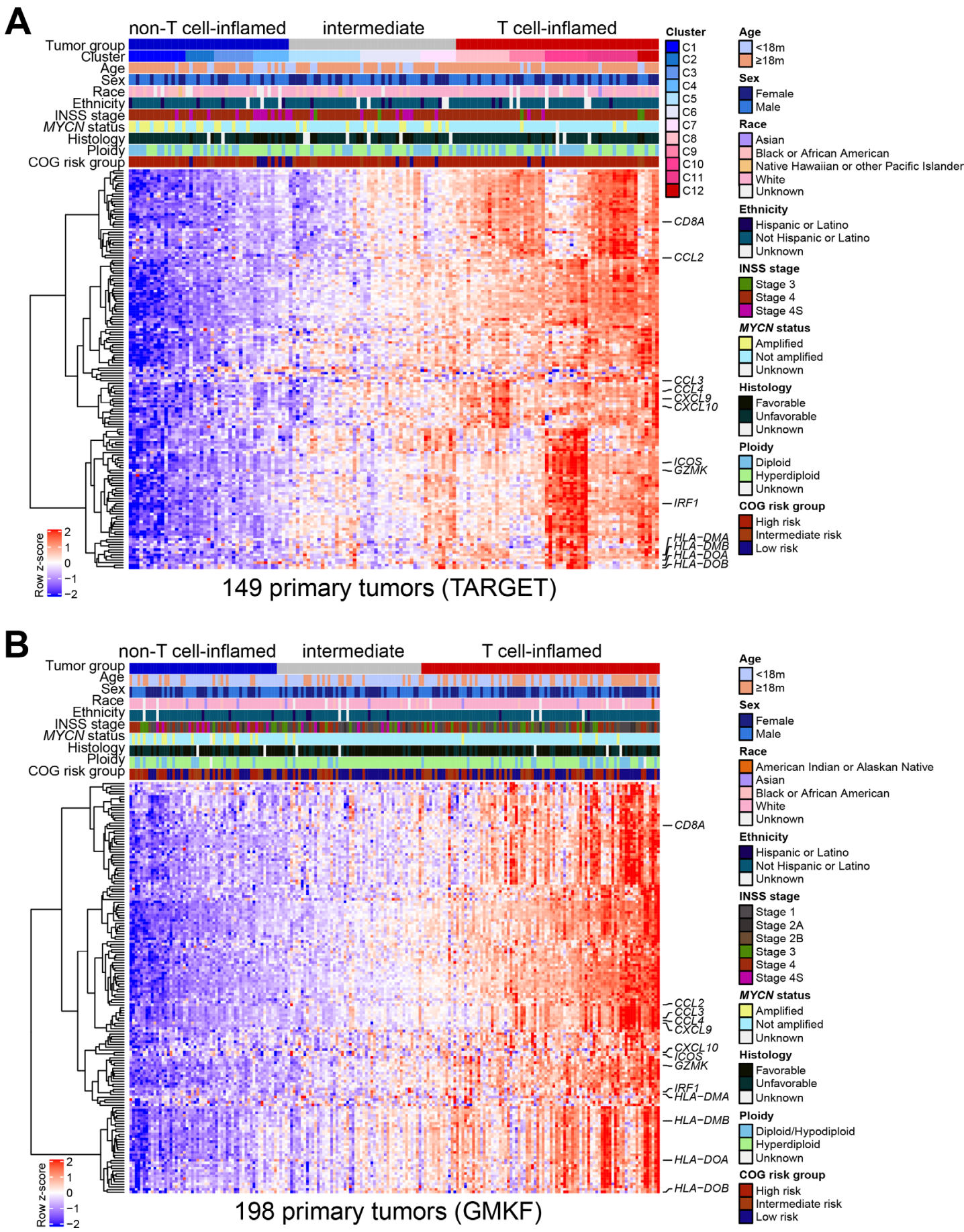

Figure 1 Immunogenomic landscape of neuroblastoma tumor microenvironment. (A) Heatmap of the T cell-inflamed gene expression signature in the discovery cohort (TARGET). Genes are on the row and tumor samples are on the column. The non-T cell-inflamed (blue), intermediate (gray), and T cell-inflamed (red) tumor groups, consensus clusters C1 to C12, MYCN amplification status, clinical and demographic factors are shown above the heatmap. $n=149$ primary tumors shown, including 123 high-risk. (B) Heatmap of the T cell-inflamed gene expression signature in the validation cohort (GMKF). Same annotation as in (A). $n=198$ primary tumors shown, including 48 high-risk. COG, Children's Oncology Group; GMKF, Gabriella Miller Kids First; TARGET, Therapeutically Applicable Research to Generate Effective Treatments.

Therefore, we combined these two groups and compared probability of survival to patients with non-T cell-inflamed tumors (OS: $\mathrm{p}=0.0076$, EFS: $\mathrm{p}=0.10$, log-rank test) $(\mathrm{KM}$ estimator shown in figure 2A,B). Similar associations between $\mathrm{T}$ cell-inflamed/intermediate tumors and improved survival were observed in the 17 high-risk patients with available survival data from the validation cohort (OS: $\mathrm{p}=0.016$, EFS: $\mathrm{p}=0.0098$ ) (figure 2C,D). No significant association with survival outcome was detected for age, MYCN status, or ploidy. Patients were not selected by diagnosis year 2010 or earlier due to small sample size. However, EFS and OS were not significantly different 
Table 1 Characteristics of patients from T cell-inflamed, intermediate, and non-T cell-inflamed tumor groups in the discovery cohort (TARGET)

\begin{tabular}{|c|c|c|c|c|}
\hline Characteristic & $\begin{array}{l}\text { T cell-inflamed } \\
\mathrm{N}=57, \text { no }(\%)\end{array}$ & $\begin{array}{l}\text { Intermediate } \\
\mathrm{N}=47, \text { no }(\%)\end{array}$ & $\begin{array}{l}\text { Non-T cell-inflamed } \\
\mathrm{N}=45, \text { no }(\%)\end{array}$ & P-value \\
\hline Age & & & & 0.088 \\
\hline$<18$ months & $6(11)$ & $11(23)$ & $12(27)$ & \\
\hline$\geq 18$ months & $51(89)$ & $36(77)$ & $33(73)$ & \\
\hline Sex & & & & 0.2 \\
\hline Female & $26(46)$ & $22(47)$ & $14(31)$ & \\
\hline Male & $31(54)$ & $25(53)$ & $31(69)$ & \\
\hline Race & & & & 0.7 \\
\hline Asian & $1(2)$ & $0(0)$ & $0(0)$ & \\
\hline Black or African American & $8(15)$ & $9(20)$ & $9(24)$ & \\
\hline $\begin{array}{l}\text { Native Hawaiian or other } \\
\text { Pacific Islander }\end{array}$ & $0(0)$ & $1(2)$ & $1(3)$ & \\
\hline White & $43(83)$ & $36(78)$ & $27(73)$ & \\
\hline Unknown & 5 & 1 & 8 & \\
\hline Ethnicity & & & & 0.5 \\
\hline Hispanic or Latino & $3(6)$ & $5(12)$ & $5(12)$ & \\
\hline Not Hispanic or Latino & $49(94)$ & $38(88)$ & $37(88)$ & \\
\hline Unknown & 5 & 4 & 3 & \\
\hline INSS stage & & & & $0.008^{* *}$ \\
\hline Stage 3 & $3(5)$ & $2(4)$ & $0(0)$ & \\
\hline Stage 4 & $52(91)$ & $37(79)$ & $34(76)$ & \\
\hline Stage 4S & $2(4)$ & $8(17)$ & $11(24)$ & \\
\hline MYCN status & & & & $<0.001^{\star \star \star}$ \\
\hline Amplified & $3(5)$ & $11(23)$ & $17(38)$ & \\
\hline Not amplified & $53(95)$ & $36(77)$ & $28(62)$ & \\
\hline Unknown & 1 & 0 & 0 & \\
\hline Histology & & & & 0.078 \\
\hline Favorable & $6(11)$ & $12(29)$ & $10(23)$ & \\
\hline Unfavorable & $48(89)$ & $29(71)$ & $33(77)$ & \\
\hline Unknown & 3 & 6 & 2 & \\
\hline Ploidy & & & & 0.4 \\
\hline Diploid & $28(50)$ & $17(36)$ & $20(44)$ & \\
\hline Hyperdiploid & $28(50)$ & $30(64)$ & $25(56)$ & \\
\hline Unknown & 1 & 0 & 0 & \\
\hline COG risk group & & & & 0.069 \\
\hline High risk & $53(92)$ & $37(79)$ & $33(73)$ & \\
\hline Intermediate risk & $2(4)$ & $6(13)$ & $5(11)$ & \\
\hline Low risk & $2(4)$ & $4(8)$ & 7 (16) & \\
\hline
\end{tabular}

$P$ values were calculated using one-way analysis of variance, $\chi^{2}$ test, Fisher's exact test.

${ }^{*} \mathrm{p}<0.05,{ }^{* \star} \mathrm{p}<0.01,{ }^{* \star *} \mathrm{p}<0.001$.

COG, Children's Oncology Group; INSS, International Neuroblastoma Staging System; TARGET, Therapeutically Applicable Research to Generate Effective Treatments.

between the 13 patients diagnosed between 2008 and 2010 and the four patients diagnosed between 2011 and 2012 (OS: $\mathrm{p}=0.98$, EFS: $\mathrm{p}=0.66$ ), and hence these are unlikely to contribute to the significantly better survival outcome observed in $\mathrm{T}$ cell-inflamed group relative to non-T cell-inflamed group in the validation cohort. 
Table 2 Characteristics of patients from T cell-inflamed, intermediate, and non-T cell-inflamed tumor groups in the validate cohort (GMKF)

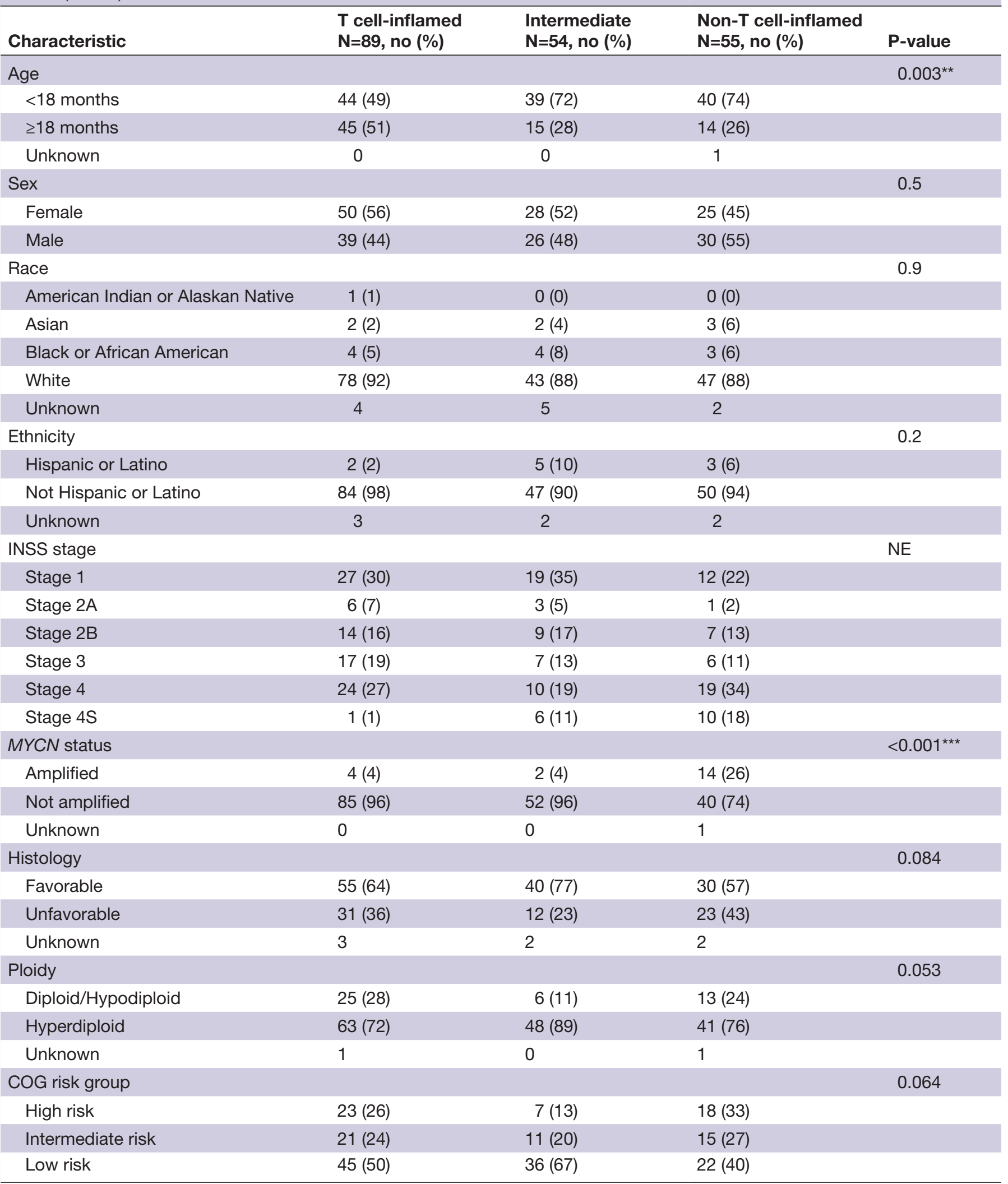

$P$ values were calculated using one-way analysis of variance, $\chi^{2}$ test, Fisher's exact test.

${ }^{*} p<0.05,{ }^{* *} p<0.01,{ }^{* * *} p<0.001$.

COG, Children's Oncology Group; GMKF, Gabriella Miller Kids First; INSS, International Neuroblastoma Staging System; NE, Not Evaluable. 
Table 3 OS and EFS of high-risk neuroblastoma patients according to T cell inflammation group and other established prognostic markers

\begin{tabular}{|c|c|c|c|c|c|c|c|c|c|}
\hline \multirow[b]{2}{*}{ Characteristic } & \multirow[b]{2}{*}{ Comparison } & \multicolumn{4}{|c|}{ Overall survival } & \multicolumn{4}{|c|}{ Event-free survival } \\
\hline & & HR & $95 \mathrm{Cl}$ low & $95 \mathrm{Cl}$ high & P-value & HR & $95 \mathrm{Cl}$ low & $95 \mathrm{Cl}$ high & P-value \\
\hline \multirow{2}{*}{$\begin{array}{l}\text { T cell } \\
\text { inflammation } \\
\text { group }\end{array}$} & $\begin{array}{l}\text { T cell-inflamed vs } \\
\text { non-T cell-inflamed }\end{array}$ & 0.558 & 0.317 & 0.982 & $0.043^{*}$ & 0.691 & 0.408 & 1.171 & 0.170 \\
\hline & $\begin{array}{l}\text { Intermediate vs } \\
\text { non-T cell-inflamed }\end{array}$ & 0.421 & 0.218 & 0.815 & $0.010^{*}$ & 0.635 & 0.353 & 1.143 & 0.130 \\
\hline MYCN status & $\begin{array}{l}\text { Amplified vs } \\
\text { not amplified }\end{array}$ & 1.281 & 0.734 & 2.236 & 0.384 & 1.155 & 0.694 & 1.923 & 0.580 \\
\hline Ploidy & $\begin{array}{l}\text { Hyperdiploid vs } \\
\text { diploid }\end{array}$ & 0.606 & 0.366 & 1.001 & 0.050 & 0.682 & 0.434 & 1.071 & 0.097 \\
\hline Age (months) & Continuous variable & 0.998 & 0.991 & 1.006 & 0.688 & 0.995 & 0.987 & 1.003 & 0.245 \\
\hline
\end{tabular}

$\mathrm{HR}$ and $\mathrm{p}$-values were calculated using Cox proportional hazards models.

${ }^{*} \mathrm{p}<0.05,{ }^{* *} \mathrm{p}<0.01,{ }^{* * *} \mathrm{p}<0.001$.

$\mathrm{Cl}$, confidence interval; EFS, event-free survival; HR, hazard ratio; OS, overall survival.

\section{Neoantigen load is a prognostic marker independent of the $\mathrm{T}$ cell-inflamed expression signature}

Neoantigens are mutant antigens that are only expressed on tumor cells and not normal cells. Neoantigen-derived epitopes (neoepitopes) are recognized by antigenspecific $\mathrm{CD}^{+}{ }^{\mathrm{T}}$ cells. ${ }^{43}$ To evaluate the neoantigen load in neuroblastoma tumors, WES data from 198 matched tumor/normal pairs of the discovery cohort carrying one
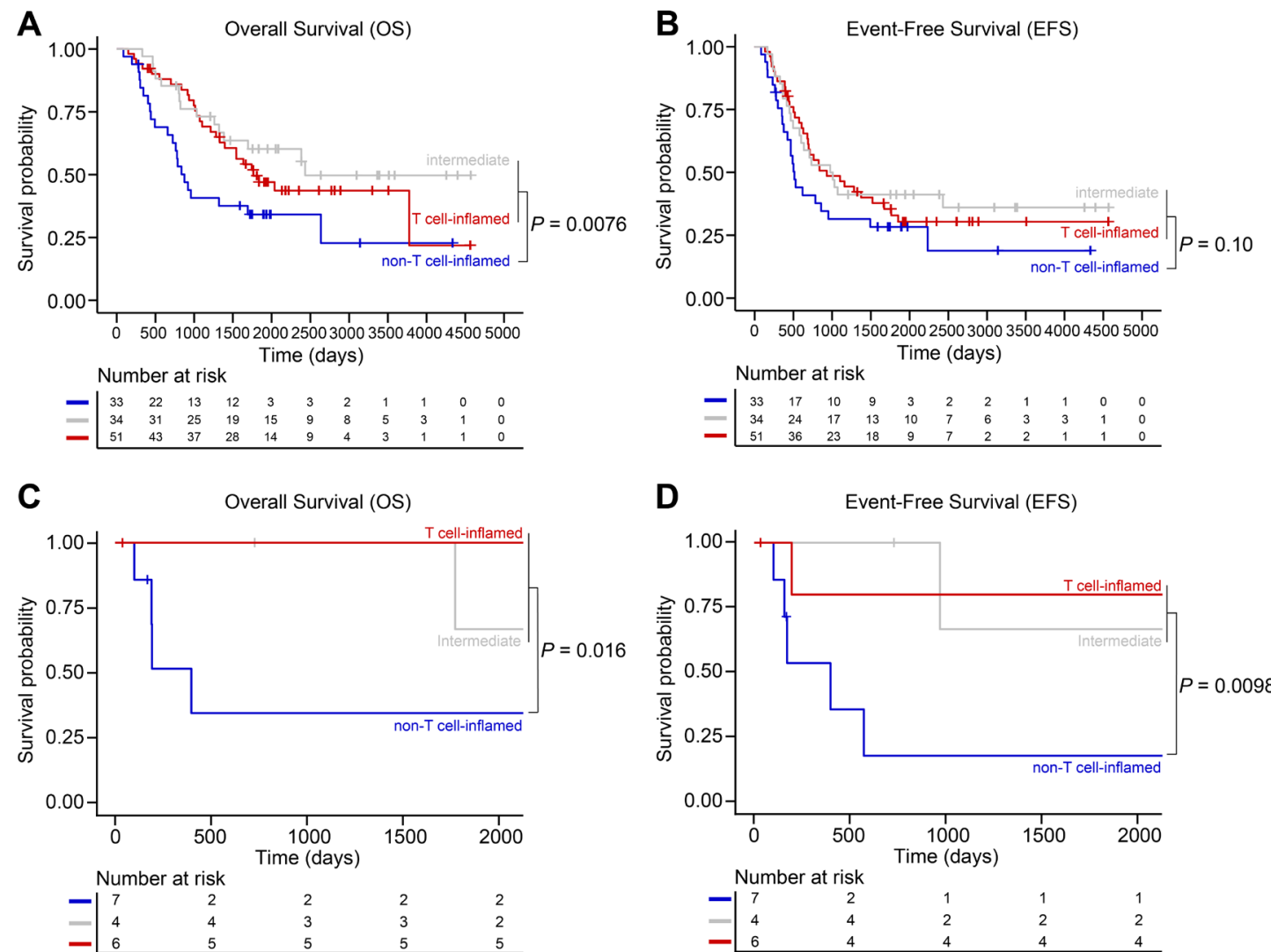

Figure 2 T cell-inflamed gene expression signature predicts overall survival in high-risk neuroblastoma patients. (A,B) KaplanMeier estimator of the T cell-inflamed (red), intermediate (gray), and non-T cell-inflamed (blue) tumor groups from the discovery cohort (TARGET), with overall survival (OS) shown in (A) and event-free survival (EFS) shown in (B). Of 123 high-risk patients, $\mathrm{n}=118$ diagnosed between year 2000 and 2010 are shown; all have survival data available. (C,D) Kaplan-Meier estimator of the T cell-inflamed (red), intermediate (gray), and non-T cell-inflamed (blue) tumor groups from the validation cohort (GMKF), with OS shown in (C) and EFS shown in (D). Of 48 high-risk patients, $n=17$ with survival data available shown. $P$-values were calculated by log-rank test in (A) to (D), comparing T cell-inflamed/intermediate tumors as one combined group to non-T cell-inflamed tumors. GMKF, Gabriella Miller Kids First; TARGET, Therapeutically Applicable Research to Generate Effective Treatments. 
or more somatic single nucleotide variants $(\mathrm{SNVs})$ were analyzed. After combining calls of four somatic callers and rigorous quality filtering, 4235 somatic SNVs were identified in 3369 genes. Each tumor harbors a median of 17 somatic SNVs (range, 1-168 SNVs), with 15 somatic SNVs predicted to alter protein sequences (range, 1-162), which is consistent with the somatic mutation profile previously reported in high-risk neuroblastoma. ${ }^{44}$ To investigate if neoantigen load was associated with outcome in high-risk patients, of 118 high-risk patients diagnosed between 2000 and 2010 in the discovery cohort, we analyzed tumors from 89 patients with both WES and RNAseq data available. The total number of neoantigens in tumor was determined by filtering for those predicted to bind to MHC class I molecule HLA-A. We focused on HLA-A molecule because the prediction algorithm for this allele is the most reliable.

A median of 4 (range 1-30) candidate neoantigens were identified in 78 of 89 tumors. Seventy-four patients diagnosed between year 2000 and 2010 were included in survival analysis. We found that the neoantigen load was significantly associated with $\mathrm{OS}(\mathrm{p}=0.00022, \log$-rank test $)$ (figure 3A) and EFS $(\mathrm{p}=0.0044)$ (figure 3B), although there was no significant difference in neoantigen load between non-T cell-inflamed and T cell-inflamed groups ( $\mathrm{p}=0.22$, two-sided Wilcoxon rank-sum test) (figure 3C). We defined four patient groups (hereafter referred as, quadrants $(\mathrm{Q})$ ) split by the threshold of $\mathrm{T}$ cell-inflamed (Tinfl) gene expression in non-T cell-inflamed tumors and median of neoantigen load (Neo) (Spearman's correlation coefficient $\rho=0.053, \mathrm{p}=0.65$ ) (figure $3 \mathrm{D}$ ): Q1 $(\mathrm{n}=8), \mathrm{Tinfl}_{\text {low }} \mathrm{Neo}_{\text {high }}$, Q2 (n=19), Tinfl low $\mathrm{Neo}_{\text {low }} ; \mathrm{Q} 3$ $(\mathrm{n}=20), \mathrm{Tinf}_{\text {high }} \mathrm{NeO}_{\text {low }} ; \mathrm{Q} 4(\mathrm{n}=27), \mathrm{Tinfl}_{\text {high }} \mathrm{NeO}_{\text {high }}$. OS and EFS were significantly different according to quadrant assignment (OS: $\mathrm{p}=0.00083$; EFS: $\mathrm{p}=0.0061$, logrank test) (figure $3 \mathrm{E}, \mathrm{F}$ ). Patients in $\mathrm{Q} 1$ and $\mathrm{Q} 4$, who had tumors harboring high level of neoantigens, had superior outcome compared with those in Q2 and Q3 (figure 3E,F).

\section{Tumor-intrinsic oncogenic transcriptional programs associated with a non-T cell-inflamed phenotype}

To investigate if tumor-intrinsic transcriptional programs may play a role in inhibiting $\mathrm{T}$ cell infiltration in non- $\mathrm{T}$ cell-inflamed neuroblastomas, we first analyzed tumors from the discovery cohort for signaling pathways intrinsic to the neoplastic cells that were previously reported to impair the local immune response in other tumor types. This includes somatic activation mutations in CTNNB1 or damaging mutations in repressors of the pathway (APC/ APC2/AXIN1/AXIN2), ${ }^{15} 17$ somatic copy number loss in PTEN or activation mutations in PIK3CA, ${ }^{18}$ activation mutations in VEGF-A, ${ }^{45}$ and loss of function mutations in B2M ${ }^{46-48}$ STK11/LKB1,${ }^{19}$ IDH1 $1 / 2,{ }^{49}$ and NRAS/KRAS/ HRAS. ${ }^{50}$ Only three tumors harbored a missense mutation in AXIN2 (p.A113T), and two tumors had PIK3CA missense (p.K111N) or nonsense mutations (p.E888X), but none occurred at the known PIK3CA activation mutation positions (AA 345, 542, 545, 546, 1043, 1044, 1047).

We next took an unbiased approach ${ }^{15-17}$ to identify transcriptional programs that are activated in non- $T$ cell-inflamed tumors by comparing the whole transcriptome RNAseq expression of 33 non-T cell-inflamed to $53 \mathrm{~T}$ cell-inflamed tumors from the high-risk patients in the discovery cohort. A total of 1730 genes were identified that were significantly differentially expressed between the two tumor groups, with 230 upregulated in non-T cell-inflamed group and 1500 upregulated in the $\mathrm{T}$ cell-inflamed group (FDR-corrected $\mathrm{p}<0.05$, fold change $\geq 1.5$ or $\leq-1.5$ ). Causal network analysis ${ }^{36}$ with Ingenuity Knowledge Base (Qiagen) identified activation of MYCN signaling in non-T cell-inflamed tumors (activation z-score $\geq 2.0, \mathrm{p}<0.05$ ), consistent with our findings showing enrichment of MYCN amplification in non-T cell-inflamed tumors (figure 4A). Immunohistochemistry (IHC) staining of a limited number of available intermediate or high-risk neuroblastoma tumors (n=17, 5 MYCN-amplified and 12 MYCN-non-amplified; images of all IHC slides are provided at https://github. com/riyuebao/NBL-TME-Immunogenomics) demonstrated lower infiltration with $\mathrm{CD}^{+} \mathrm{T}$ cell and $\mathrm{Batf3}^{+}$ DCs in MYCN-amplified tumors compared with $M Y C N$ non-amplified neuroblastomas (figure 4B), although the difference did not reach statistical significance in this small cohort ( $\mathrm{p}=0.22$, two-sided Fisher's exact test). In both the discovery and validation cohorts, the DC genes are highly correlated with the T cell-inflamed gene expression ( $<<0.05$, Spearman's correlation) (online supplemental figure 1).

To determine if activation of transcriptional programs other than MYCN signaling is associated with the non-T cell-inflamed phenotype, we repeated the differential gene expression and causal network analyses using only MYCN non-amplified tumors $(\mathrm{n}=91)$. Genes significantly upregulated in 16 non-T cell-inflamed neuroblastomas compared with $49 \mathrm{~T}$ cell-inflamed tumors were used to predict upstream regulators. Three pathways (ASCL1, SOX11, and KMT2A) were identified to be activated in non-T cell-inflamed tumors without $M Y C N$ amplification (activation $\mathrm{z}$-score $\geq 2.0, \mathrm{p}<0.05$ ) (figure $4 \mathrm{C}$ ). We next calculated an activation score for each pathway using previously described methods. ${ }^{17} 21$ The results showed that the three pathways operate in a partially exclusive manner (online supplemental figure 2), with activation of SOX11, KMT2A, and ASCL1 signaling detected in 66\%, $30 \%$, and $30 \%$ of non-T cell-inflamed tumors, respectively, compared with less than $5 \%$ of the T cell-inflamed tumors (figure 4D,E). Taking together, the activation of one or more pathways regulated by MYCN, ASCL1, SOX11, or KMT2A was found in $85 \%$ of the non-T cell-inflamed tumors. The inverse correlation between the expression of T cell-inflamed gene signature and the four pathways (MYCN, ASCL1, SOX11, KMT2A) was confirmed in the validation cohort (online supplemental figure 3A,B), providing strong evidence that the activation of the four 


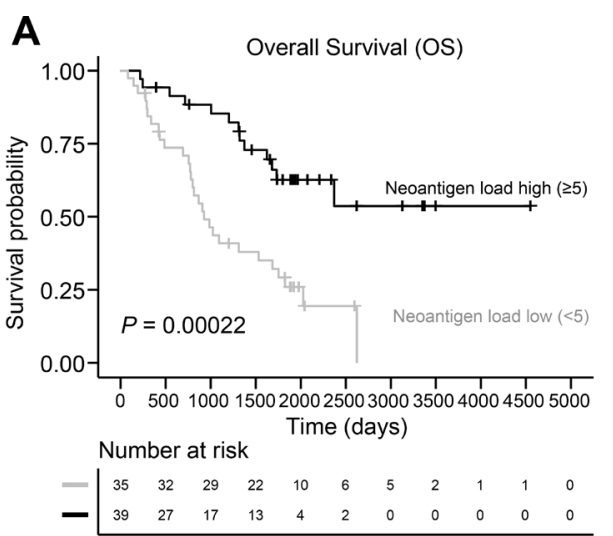

C
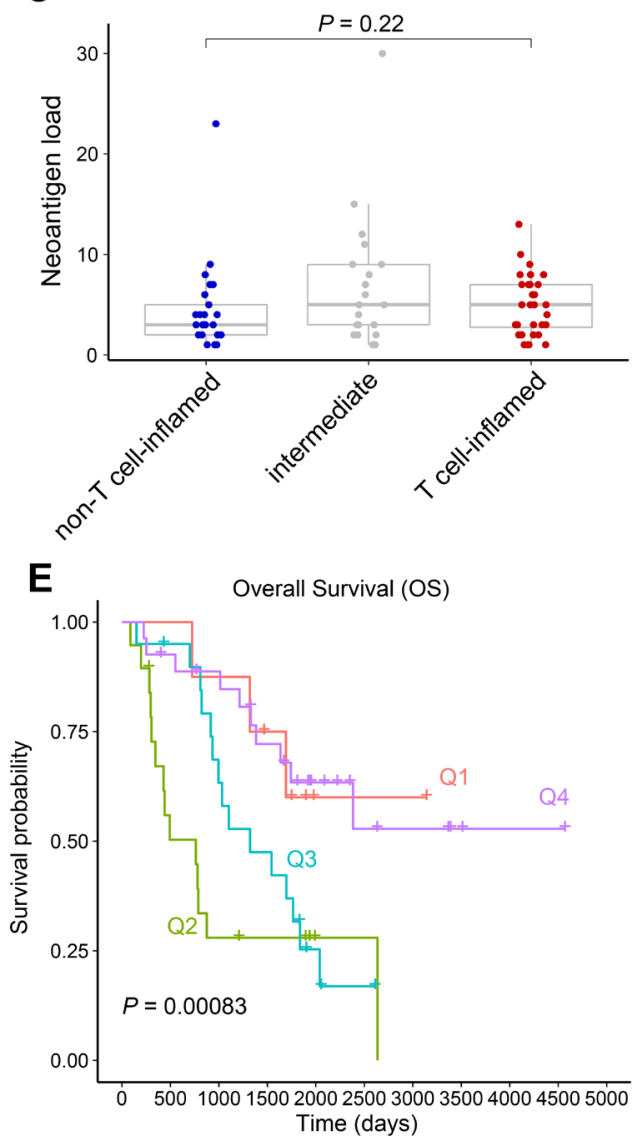

Number at risk

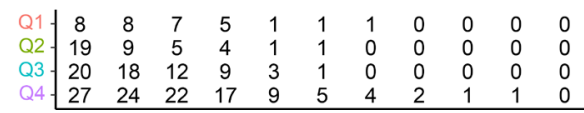

B

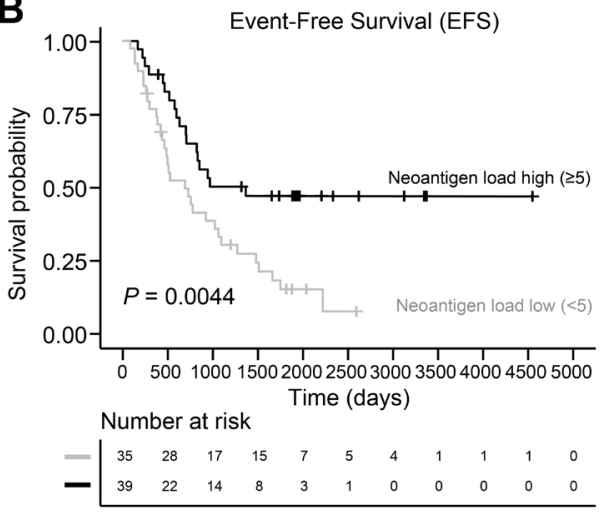

$\begin{array}{ll}\text { : non-T cell-inflamed } & \text { Spearman's } \rho=0.053 \\ \text { : intell-inflamed } & P=0.65\end{array}$

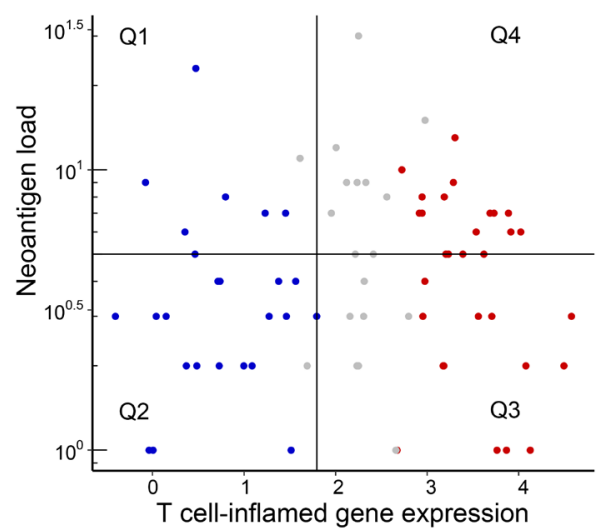

$\mathbf{F}$

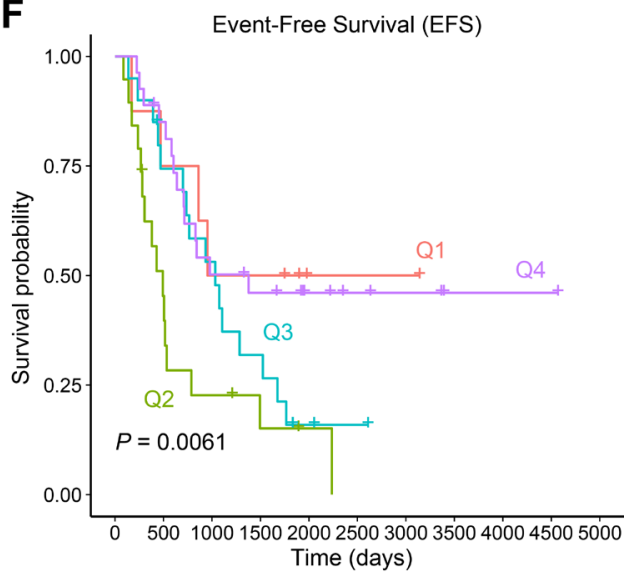

Number at risk

\begin{tabular}{l|ccccccccccc} 
Q1 & -8 & 6 & 4 & 4 & 1 & 1 & 1 & 0 & 0 & 0 & 0 \\
Q3 & 19 & 8 & 4 & 2 & 1 & 0 & 0 & 0 & 0 & 0 & 0 \\
24 & 14 & 10 & 6 & 2 & 1 & 0 & 0 & 0 & 0 & 0 \\
27 & 22 & 13 & 11 & 6 & 4 & 3 & 1 & 1 & 1 & 0 \\
\hline
\end{tabular}

Figure 3 Neoantigen load predicts overall and event-free survival in high-risk neuroblastoma patients. (A,B) Kaplan-Meier estimator of the neoantigen load high and low tumor groups in association with overall survival (OS) shown in (A) and eventfree survival (EFS) shown in (B). (C) Neoantigen load between non-T cell-inflamed, intermediate, and T cell-inflamed groups. (D) Quadrants (Q1 to Q4) according to expression of T cell-inflamed gene signature (x-axis) and the neoantigen load (y-axis). Vertical line labels the separation of non-T cell-inflamed group versus intermediate or T cell-inflamed group, and horizontal line labels the median of neoantigen load across samples. (E,F) Kaplan-Meier estimator of the four groups from (D), with OS shown in (E) and EFS shown in (F). $n=78$ patients from the discovery cohort (TARGET) with both WES and RNAseq data available and having at least one candidate neoepitope detected are shown in (C). $n=74$ patients diagnosed between year 2000 and 2010 are shown in (A, B, D, E, and F). P-values were calculated by two-sided Wilcoxon rank-sum test in (C), Spearman's correlation in (D), and log-rank test in (A, B, E, and F). TARGET, Therapeutically Applicable Research to Generate Effective Treatments; WES, whole-exome sequencing. 
A

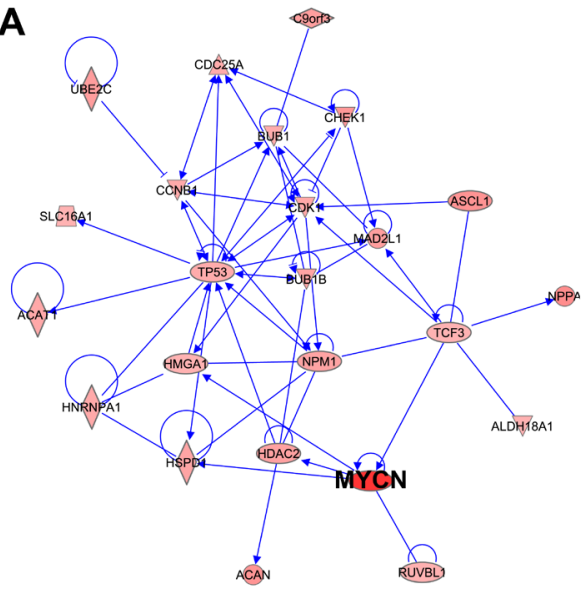

B
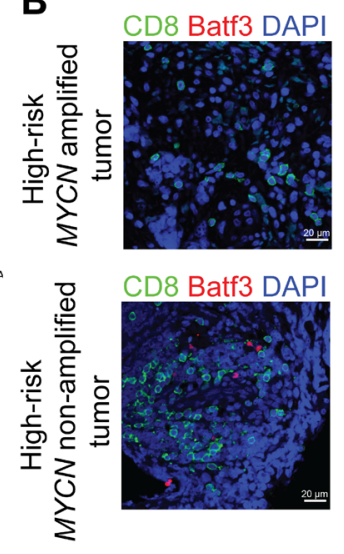

C

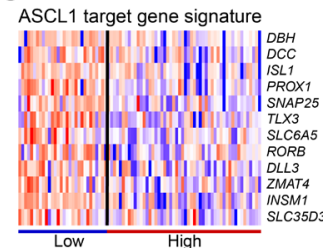

OX11 target gene signature

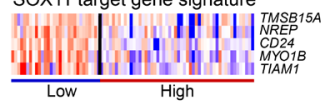

KMT2A target gene signature

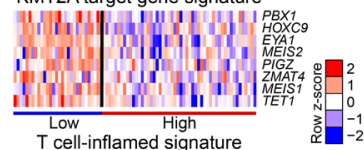

Toll-inflamed signature

T cell-inflamed

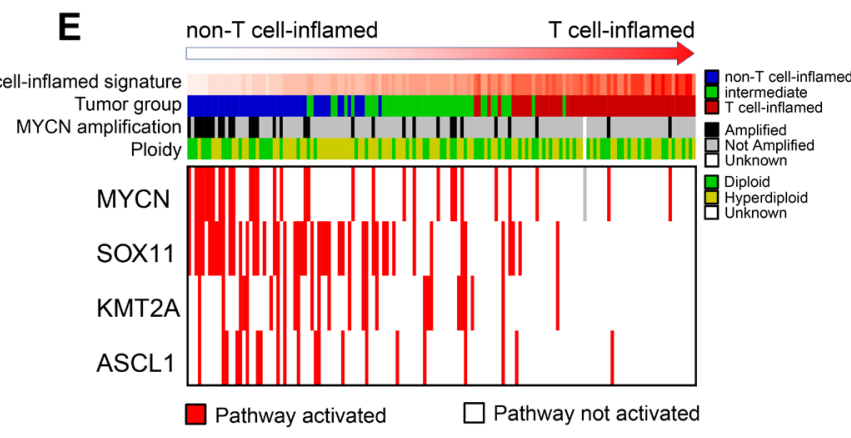

Figure 4 Neuroblastoma-intrinsic oncogenic pathway activation correlates with the non-T cell-inflamed tumor microenvironment. (A) Activation of MYCN signaling network. Arrows indicate direction (from the upstream regulator to the downstream target molecules). Pink color indicates the gene is upregulated in non-T cell-inflamed tumors relative to inflamed. (B) Immunofluorescent IHC straining against $\mathrm{CD} 8^{+} \mathrm{T}$ cells and Batf3 ${ }^{+} \mathrm{DCs}$ in $\mathrm{MYCN}$-amplified and non-amplified tumors. Scale bars, $20 \mu \mathrm{m}$. (C) Target molecule expression heatmap of ASCL1, SOX11, and KMT2A as upstream regulators in non-T cell-inflamed versus T cell-inflamed tumors. (D) The percentage of tumors harboring activation in MYCN, ASCL1, SOX11, and KMT2A transcriptional programs (defined as, pathway activation score $>0.5^{17}$ ) in non-T cell-inflamed and T cell-inflamed groups. An aggregation of the four pathways (MYCN, ASCL1, SOX11, and KMT2A) is shown on top. (E) Correlation between the T cellinflamed gene signature and activation of each pathway at a continuous scale. Tumor samples were sorted by $T$ cell-inflamed gene expression (left to right: lower to higher).

transcriptional programs was significantly associated with a non-T cell-inflamed phenotype.

\section{DISCUSSION}

Although improved survival and response to immunotherapy have been observed in adults with cancers showing $\mathrm{T}$ cell infiltration, the immunobiology of neuroblastoma tumors and its association with outcome had been poorly understood. In this study, we categorized 149 clinically annotated primary neuroblastoma tumors in the TARGET program as $\mathrm{T}$ cell-inflamed, non- $\mathrm{T}$ cell-inflamed, and intermediate using a defined $\mathrm{T}$ cellinflamed gene expression signature. The gene signature also identified the same three tumor groups in an independent cohort comprised of publicly available tumor genomic data housed in the GMKF program linked to clinical information in the INRG Data Commons. In both cohorts, MYCN amplification was significantly more prevalent in the non-T cell-inflamed tumors compared with the $\mathrm{T}$ cell-inflamed tumors. Interestingly, we also found that patients in both cohorts diagnosed at age $<18$ months had tumors that were enriched in the non-T cell-inflamed tumor group.

In analyses restricted to high-risk patients in the TARGET cohort, OS was significantly better for those with $\mathrm{T}$ cell-inflamed tumors compared with those with non-T cell-inflamed tumors. A similar trend was observed for EFS, although statistical significance was not reached. Further, the T cell-inflamed signature maintained independent statistical significance for OS in multivariable analysis adjusted for age, MYCN status, and ploidy. This association between $\mathrm{T}$ cell-inflamed tumors and superior outcome was validated in the clinically annotated GMKF cohort of tumors. Because neoantigens are recognized by the immune system and can be targeted to increase anti-tumor immunity, ${ }^{43}$ we also analyzed neoantigen load in the neuroblastoma tumors. Although no significant difference in neoantigen load was detected among $\mathrm{T}$ cell-inflamed, non-T cell-inflamed, or intermediate groups, superior OS was seen in the cohort of patients with tumors harboring a high neoantigen load. Taken together, these results suggest that $\mathrm{T}$ cell-inflamed gene expression and high neoantigen load may independently 
impact the clinical behavior of neuroblastoma tumors, resulting in improved survival.

The lack of correlation between the T cell-inflamed expres-

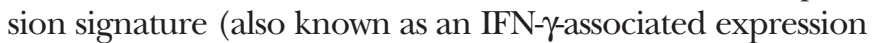
signature) and TMB, which is highly correlated with neoantigen load, ${ }^{51}$ has been reported in many adult cancers, including melanoma, ${ }^{13}$ head and neck, ${ }^{7}$ and pan-cancer. ${ }^{52}$ It is well established in the literature that TMB (or neoantigen load) and $\mathrm{T}$ cell-inflamed expression are both prognostic but seemly have little correlation. ${ }^{10-12}$ In particular, the pan-cancer study reports four groups of patients determined by high/low IFN- $\gamma$-associated expression signature and high/low TMB ${ }^{52}$ Only patients possessing high levels of both signatures had the best response rate, and a significant number of patients only showed high levels of one of the signatures. ${ }^{52}$ The mechanism underlying the decoupling of T cell-inflamed expression signature and TMB and neoantigen load remains to be explored.

Others have evaluated inflammatory cell infiltrates in neuroblastoma tumors using different methodologies and markers. Asgharzadeh and colleagues ${ }^{53}$ assessed the relationship between TAMs and the clinical behavior of metastatic MYCN-non-amplified neuroblastoma. Using IHC, significantly greater numbers of infiltrating macrophages with CD163 staining, which identifies alternatively activated M2 macrophages, were observed in metastatic neuroblastomas compared with locoregional tumors. Thus, TAMs may promote aggressive growth in neuroblastoma, as reported in Hodgkin's lymphoma ${ }^{54}$ and breast cancer. ${ }^{55}$ Further, expression studies using a TaqMan low-density array assay demonstrated higher levels of inflammation-related genes (CD14, CD33, FCGR3 (CD16), interleukin-6 receptor, and interleukin-10) in tumors from patients diagnosed at $\geq 18$ months compared with younger patients. These inflammatory genes are largely expressed in macrophages and can signify intratumor macrophage polarization to the anti-inflammatory M2-like phenotype, suggesting that TAMs contribute to the aggressive clinical behavior of neuroblastomas associated with older age. Age is an established prognostic marker in neuroblastoma, and more favorable outcome is associated with age $<18$ months, reflecting the unique biology of infant tumors. ${ }^{40}$ Although different inflammatory cells were evaluated in our studies, we observed a higher prevalence of $\mathrm{T}$ cell-inflamed tumors in patients diagnosed $\geq 18$ months compared with infants. While specific inflammatory cells differentially influence tumor growth, the age-dependent differences in expression of tumor-associated inflammatory cell genes may contribute to underlying favorable tumor phenotype that is commonly seen in infants with neuroblastoma.

More recently, Wei and colleagues ${ }^{56}$ analyzed gene expression signatures of TILs in neuroblastomas, immune cells that have previously been reported to be predictive of clinical outcomes for patients with cancers. ${ }^{57}$ Similar to our study, higher levels of cytotoxic TIL signature genes were observed in the MYCN-non-amplified tumors compared with tumors with amplification of MYCN.
Further, these investigators also reported improved survival in a cohort of patients with $M Y C N$-non-amplified tumors with increased signatures for activated NK cells, $\mathrm{CD}^{+} \mathrm{T}$ cells, cytolytic activity, clonal expansion of $\mathrm{T}$ cell receptors, and exhaustion markers.

The inverse correlation between MYCN amplification and T cell-inflamed tumors seen in our study and others ${ }^{5356}$ suggests that MYCN signaling inhibits $\mathrm{T}$ cell infiltration in neuroblastoma tumors. In support of MYCN's role in mediating exclusion of $\mathrm{T}$ cells from the microenvironment of neuroblastoma tumors, we identified activation of MYCN signaling in non-T cell-inflamed tumors (activation $\mathrm{z}$-score $\geq 2.0, \mathrm{p}<0.05$ ) comparing expression profiles between non-T cell-inflamed and T cell-inflamed tumors. In addition, we identified three transcriptional programs, ASCL1, SOX11, and KMT2A, that were activated in non-T cell-inflamed tumors without MYCN amplification.

ASCL1 (alias hASH1 in human) is a known proneural transcription factor essential for neurogenesis. However, in neuroblastoma ASCL1 represses genes involved in neuron differentiation. ${ }^{58}$ Recent studies have demonstrated that ASCL1 is a MYCN-dependent and LMO1dependent member of the adrenergic neuroblastoma core regulatory circuitry (CRC), an interconnected autoregulatory loop of transcription factors whose expression is driven by themselves and other members of the CRC. ${ }^{59}$ Interestingly, LMO1 and the CRC members bind to enhancer elements and directly upregulate the ASCL1 gene, resulting in promotion of cell growth and repression of neuronal differentiation. ${ }^{59}$ Activation of ASCL1 signaling is also predictive of poor prognosis in neuroendocrine lung cancers. ${ }^{60}$ In glioblastoma, ASCL1 is critical to the maintenance of stem cells through activation of WNT signaling. ${ }^{61}$ SOX11 is a transcription factor essential for neuron survival and neurite outgrowth. ${ }^{62}$ In our study, the expression of MYCN and SOX11 pathways is positively correlated (Spearman's $\rho=0.81$ in TARGET and 0.83 in GMKF, respectively, $\mathrm{p}<0.0001$ ), suggesting the two mechanisms may interact. Indeed, recent studies reported that SOX11 was a direct target of MYCN. ${ }^{63}$ However, $30 \%$ of MYCN non-amplified tumors showed SOX11 pathway activation, which may indicate other signaling routes independent of MYCN. KMT2A (alias MLL1 in human) is an epigenetic regulator of neuronal function. In pancreatic cancer where anti-PD1/PD-L1 immunotherapy is ineffective, MLL1 directly binds to the promoter of the checkpoint inhibitor $P D-L 1$ and activates its transcription, and combinatorial therapy of anti-MLL1 and anti-PD1/PD-L1 was proven to suppress tumor growth in mouse models. ${ }^{64}$ Taken together, these transcriptional programs support a stem cell-like phenotype in neural tissues, which is a consistent theme with what has been observed in adult tumors for a state of epithelial-mesenchymal transition being associated with immuno-oncology resistance. ${ }^{65}$

In conclusion, the association of improved survival with T cell-inflamed neuroblastoma and high neoantigen load indicate that crosstalk between tumor cells and components of the microenvironment influence neuroblastoma 
phenotype. Our studies also suggest that tumor-intrinsic MYCN, ASCL1, SOX11, or KMT2A signaling may mediate immune exclusion in neuroblastoma. Understanding the molecular mechanisms that drive the presence or absence of $\mathrm{T}$ cell infiltration and neoantigen load should enable more personalized treatment approaches and provide insight for the development of new therapies that may enhance response to immunotherapy and improve outcome. Clinical trials testing the efficacy of anti-GD2 antibodies and other modalities of immunotherapy in patients with neuroblastoma tumors that are $\mathrm{T}$ cellinflamed or harbor high neoantigen load are warranted.

\section{Author affiliations}

${ }^{1}$ Hillman Cancer Center, University of Pittsburgh Medical Center, Pittsburgh, Pennsylvania, USA

${ }^{2}$ Department of Medicine, University of Pittsburgh, Pittsburgh, Pennsylvania, USA ${ }^{3}$ Koch Institute for Integrative Cancer Research at MIT, Massachusetts Institute of Technology, Cambridge, Massachusetts, USA

${ }^{4}$ Department of Biology, Massachusetts Institute of Technology, Cambridge, Massachusetts, USA

${ }^{5}$ Center for Translational Data Science, The University of Chicago, Chicago, Illinois, USA

${ }^{6}$ Department of Medicine, The University of Chicago, Chicago, Illinois, USA

${ }^{7}$ Department of Pathology, The University of Chicago, Chicago, Illinois, USA

${ }^{8}$ Department of Pediatrics, The University of Chicago, Chicago, Illinois, USA

Twitter Riyue Bao @RiyueSunnyBao and Jason J Luke @jasonlukemd

Acknowledgements The bioinformatics analysis was performed on the highperformance computing (HPC) cluster Gardner at Center for Research Informatics (U. Chicago) and on the HPC cluster HTC at Center for Research Computing (U. Pittsburgh). The authors thank M Jarsulic (U. Chicago) and F Mu (U. Pittsburgh) for their technical assistance in software installation and job execution on the HPCs.

Contributors RB, SLC, and AVD conceived the study. AVD supervised the project. $\mathrm{RB}$ acquired the data, developed the methodology, performed the computations, and analyzed the data. KH performed the neoantigen prediction and filtering. YZ performed the $\mathrm{IHC}$ staining and scanned the slides. PP examined the pathology slides. RB, SS, JJL, TFG, SLC, and AVD interpreted the results. RB, SS, SLC, and AVD wrote the manuscript. All authors contributed to the final manuscript.

Funding RB and JJL acknowledge funding from the Hillman Fellows for Innovative Cancer Research Program. SS was a postdoctoral fellow of the Cancer Research Institute. JJL: Department of Defense Career Development Award (W81XWH-171-0265), the Arthur J Schreiner Family Melanoma Research Fund, the J Edward Mahoney Foundation Research Fund, Brush Family Immunotherapy Research Fund and Buffet Fund for Cancer Immunotherapy. TG: American Cancer Society-Jules L Plangere Jr. Family Foundation Professorship in Cancer Immunotherapy, and R35 CA210098 from the NIH. The INRG database is supported in part by the William Guy Forbeck Research Foundation, the St Baldrick's Foundation, the Little Heroes Cancer Research Fund, Children's Neuroblastoma Cancer Foundation, Neuroblastoma Children's Cancer Foundation, the Super Jake Foundation, The Matthew Bittker Foundation, and the Alex's Lemonade Stand Foundation. Data included in the INRG database were provided by Children's Oncology Group (COG), Pediatric Oncology Group (POG), Children's Cancer Study Group (CCSG), German Gesellschaft für Pädiatrische Onkologie und Hämatologie (GPOH), European Neuroblastoma Study Group (ENSG), International Society of Paediatric Oncology Europe Neuroblastoma Group (SIOPEN), Japanese Neuroblastoma Study Group (JNBSG), Japanese Infantile Neuroblastoma Co-operative Study Group (JINCS), Spanish Neuroblastoma Group and the Italian Neuroblastoma Group.

Competing interests RB declares patents: (all provisional) PCT/US15/612657 (Cancer Immunotherapy), PCT/US18/36052 (Microbiome Biomarkers for Anti-PD-1/ PD-L1 Responsiveness: Diagnostic, Prognostic and Therapeutic Uses Thereof), PCT/ US63/055227 (Methods and Compositions for Treating Autoimmune and Allergic Disorders); JJL declares Data and Safety Monitoring Board: TTC Oncology, Scientific Advisory Board: 7 Hills, Actym, Alphamab Oncology, Array, BeneVir, Mavu, Tempest, Consultancy: Aduro, Astellas, AstraZeneca, Bayer, Bristol-Myers Squibb, Castle, CheckMate, Compugen, EMD Serono, IDEAYA, Immunocore, Janssen, Jounce, Leap,
Merck, Mersana, NewLink, Novartis, RefleXion, Spring Bank, Syndax, Tempest, Vividion, WntRx, Research Support: (all to institution for clinical trials unless noted) AbbVie, Array (Scientific Research Agreement; SRA), Boston Biomedical, Bristol-Myers Squibb, Celldex, CheckMate (SRA), Compugen, Corvus, EMD Serono, Evelo (SRA), Delcath, Five Prime, FLX Bio, Genentech, Immunocore, Incyte, Leap, Medlmmune, Macrogenics, Novartis, Pharmacyclics, Palleon (SRA), Merck, Tesaro, Xencor, Travel: Array, AstraZeneca, Bayer, BeneVir, Bristol-Myers Squibb, Castle, CheckMate, EMD Serono, IDEAYA, Immunocore, Janssen, Jounce, Merck, Mersana, NewLink, Novartis, RefleXion, Patents: (both provisional) Serial \#15/612,657 (Cancer Immunotherapy), PCT/US18/36052 (Microbiome Biomarkers for Anti-PD-1/PD-L1 Responsiveness: Diagnostic, Prognostic and Therapeutic Uses Thereof). SS declares a patent on WNT/ $\beta$-catenin targeting to enhance anti-tumor immune responses (PCT15/155,099), serves on the SAB on Venn Therapeutics, Tango Therapeutics, Arcus Biosciences and consults for TAKEDA, Replimune, Ribon, Dragonfly and Merck. TFG has received consultancy fees from Merck, Roche-Genentech, Abbvie, Bayer, Jounce, Aduro, Fog Pharma, Adaptimmune, FivePrime, and Sanofi. TFG has received research support from Roche-Genentech, BMS, Merck, Incyte, Seattle Genetics, Celldex, Ono, Evelo, Bayer, Aduro. TFG has intellectual property/licensing agreements with Aduro, Evelo, and BMS. TFG is a co-founder/shareholder of Jounce and Pyxis Oncology. AVD declares Research funding (all to institution for clinical trials): Merck, Roche, Jubilant Draxlmage, YMabs, GlaxoSmithKline, Actuate Therapeutics, Lilly; Scientific Advisory Board: Merck; Consultancy: Ology Medical Education; Travel/Accommodations: GlaxoSmithKline; Stock: Pfizer (all outside the submitted work). SLC declares Research funding (to the institution for clinical trials): Merck and United Therapeutics; Stock (personal or immediate family member): United Therapeutics, Merck, Stryker, Amgen, Pfizer, AbbVie, Jazz Pharmaceuticals, Lilly, Sanofi, Varex Imaging, Accelerated Medical Diagnostics, Anthem, Cardinal Health, Novo Nordisk, Regeneron, Zimmer BioMet (all outside the submitted work). The remaining authors declare no competing interests.

Patient consent for publication Not required.

Ethics approval Each country or cooperative group submitting data from clinical trials to the INRG Data Commons obtained institutional review board approval and informed patient consent for their respective studies. The INRG Data Commons has approval from The University of Chicago Institutional Review Board.

Provenance and peer review Not commissioned; externally peer reviewed.

Data availability statement All data relevant to the study are included in the article or uploaded as supplementary information. The gene expression, somatic mutations, and clinical data were downloaded from GDC data portal (https://portal. gdc.cancer.gov), and GMKF data portal (https://portal.kidsfirstdrc.org/dashboard). Patient-level clinical data of the GMKF cohort are under controlled access at INRG Data Commons and can be requested by contacting the INRG Review Committee (https://inrgdb.org). IHC image data files were deposited on a publicly accessible GitHub Repository (https://github.com/riyuebao/NBL-TME-Immunogenomics). Other data will be provided upon request from the corresponding author.

Supplemental material This content has been supplied by the author(s). It has not been vetted by BMJ Publishing Group Limited (BMJ) and may not have been peer-reviewed. Any opinions or recommendations discussed are solely those of the author(s) and are not endorsed by BMJ. BMJ disclaims all liability and responsibility arising from any reliance placed on the content. Where the content includes any translated material, BMJ does not warrant the accuracy and reliability of the translations (including but not limited to local regulations, clinical guidelines, terminology, drug names and drug dosages), and is not responsible for any error and/or omissions arising from translation and adaptation or otherwise.

Open access This is an open access article distributed in accordance with the Creative Commons Attribution Non Commercial (CC BY-NC 4.0) license, which permits others to distribute, remix, adapt, build upon this work non-commercially, and license their derivative works on different terms, provided the original work is properly cited, appropriate credit is given, any changes made indicated, and the use is non-commercial. See http://creativecommons.org/licenses/by-nc/4.0/.

\section{ORCID iDs}

Riyue Bao http://orcid.org/0000-0002-6105-1704

Jason J Luke http://orcid.org/0000-0002-1182-4908

\section{REFERENCES}

1 Galon J, Costes A, Sanchez-Cabo F, et al. Type, density, and location of immune cells within human colorectal tumors predict clinical outcome. Science 2006;313:1960-4. 
2 Azimi F, Scolyer RA, Rumcheva P, et al. Tumor-Infiltrating lymphocyte grade is an independent predictor of sentinel lymph node status and survival in patients with cutaneous melanoma. $J$ Clin Oncol 2012;30:2678-83.

3 Mahmoud SMA, Paish EC, Powe DG, et al. Tumor-Infiltrating CD8+ lymphocytes predict clinical outcome in breast cancer. $J$ Clin Oncol 2011;29:1949-55.

4 Spranger S, Spaapen RM, Zha Y, et al. Up-regulation of PD-L1, IDO, and $\mathrm{T}$ (regs) in the melanoma tumor microenvironment is driven by CD8(+) T cells. Sci Trans/ Med 2013;5:200ra116.

5 Taube JM, Anders RA, Young GD, et al. Colocalization of inflammatory response with B7-h1 expression in human melanocytic lesions supports an adaptive resistance mechanism of immune escape. Sci Transl Med 2012;4:127ra137.

6 Tumeh PC, Harview CL, Yearley JH, et al. PD-1 blockade induces responses by inhibiting adaptive immune resistance. Nature 2014;515:568-71.

7 Ayers M, Lunceford J, Nebozhyn M, et al. IFN- $\gamma$-related mRNA profile predicts clinical response to PD-1 blockade. J Clin Invest 2017;127:2930-40.

8 Ji R-R, Chasalow SD, Wang L, et al. An immune-active tumor microenvironment favors clinical response to ipilimumab. Cancer Immunol Immunother 2012;61:1019-31.

9 Trujillo JA, Sweis RF, Bao R, et al. T Cell-Inflamed versus non-T CellInflamed tumors: a conceptual framework for cancer immunotherapy drug development and combination therapy selection. Cancer Immunol Res 2018;6:990-1000.

10 Cristescu R, Mogg R, Ayers M, et al. Pan-tumor genomic biomarkers for PD-1 checkpoint blockade-based immunotherapy. Science 2018;362:eaar3593.

11 Rizvi NA, Hellmann MD, Snyder A, et al. Cancer immunology. Mutational landscape determines sensitivity to PD-1 blockade in non-small cell lung cancer. Science 2015;348:124-8.

12 Liu D, Schilling B, Liu D, et al. Integrative molecular and clinical modeling of clinical outcomes to PD1 blockade in patients with metastatic melanoma. Nat Med 2019;25:1916-27.

13 Spranger S, Luke JJ, Bao R, et al. Density of immunogenic antigens does not explain the presence or absence of the T-cell-inflamed tumor microenvironment in melanoma. Proc Natl Acad Sci U S A 2016;113:E7759-68.

14 Litchfield K, Reading JL, Puttick C, et al. Meta-Analysis of tumor- and T cell-intrinsic mechanisms of sensitization to checkpoint inhibition. Cell 2021;184:e514:596-614.

15 Spranger S, Bao R, Gajewski TF. Melanoma-intrinsic $\beta$-catenin signalling prevents anti-tumour immunity. Nature 2015;523:231-5.

16 Sweis RF, Spranger S, Bao R, et al. Molecular drivers of the Non-Tcell-Inflamed tumor microenvironment in urothelial bladder cancer. Cancer Immunol Res 2016;4:563-8.

17 Luke JJ, Bao R, Sweis RF, et al. WNT/B-catenin Pathway Activation Correlates with Immune Exclusion across Human Cancers. Clin Cancer Res 2019;25:3074-3083.

18 Peng W, Chen JQ, Liu C, et al. Loss of PTEN promotes resistance to T cell-mediated immunotherapy. Cancer Discov 2016;6:202-16.

19 Skoulidis F, Goldberg ME, Greenawalt DM, et al. STK11/LKB1 Mutations and PD-1 Inhibitor Resistance in KRAS-Mutant Lung Adenocarcinoma. Cancer Discov 2018;8:822-35.

20 Casey SC, Tong L, Li Y, et al. MYC regulates the antitumor immune response through CD47 and PD-L1. Science 2016;352:227-31.

21 Bao R, Stapor D, Luke JJ. Molecular correlates and therapeutic targets in T cell-inflamed versus non-T cell-inflamed tumors across cancer types. Genome Med 2020;12:90.

22 Lawrence MS, Stojanov P, Polak P, et al. Mutational heterogeneity in cancer and the search for new cancer-associated genes. Nature 2013;499:214-8.

23 Park JA, Cheung N-KV. Limitations and opportunities for immune checkpoint inhibitors in pediatric malignancies. Cancer Treat Rev 2017;58:22-33.

24 Geoerger B, Kang HJ, Yalon-Oren M, et al. Pembrolizumab in paediatric patients with advanced melanoma or a PD-L1-positive, advanced, relapsed, or refractory solid tumour or lymphoma (KEYNOTE-051): interim analysis of an open-label, single-arm, phase 1-2 trial. Lancet Oncol 2020;21:121-33.

25 Davis KL, Fox E, Merchant MS, et al. Nivolumab in children and young adults with relapsed or refractory solid tumours or lymphoma (ADVL1412): a multicentre, open-label, single-arm, phase 1-2 trial. Lancet Oncol 2020;21:541-50.

26 Yu AL, Gilman AL, Ozkaynak MF, et al. Anti-GD2 antibody with GMCSF, interleukin-2, and isotretinoin for neuroblastoma. N Engl J Med 2010;363:1324-34

27 Furman WL, Federico SM, McCarville MB, et al. A phase II trial of Hu14.18K322A in combination with induction chemotherapy in children with newly diagnosed high-risk neuroblastoma. Clin Cancer Res 2019;25:6320-8.

28 Mody R, Naranjo A, Van Ryn C, et al. Irinotecan-temozolomide with temsirolimus or dinutuximab in children with refractory or relapsed neuroblastoma (COG ANBL1221): an open-label, randomised, phase 2 trial. Lancet Oncol 2017;18:946-57.

29 Mody R, Yu AL, Naranjo A, et al. Irinotecan, temozolomide, and Dinutuximab with GM-CSF in children with refractory or relapsed neuroblastoma: a report from the children's Oncology Group. J Clin Oncol 2020;38:2160-9.

30 Zhang Z, Hernandez K, Savage J, et al. Uniform genomic data analysis in the $\mathrm{NCl}$ genomic data commons. Nat Commun 2021;12:1226.

31 Volchenboum SL, Cox SM, Heath A, et al. Data commons to support pediatric cancer research. Am Soc Clin Oncol Educ Book 2017;37:746-52.

32 Andrews S. FastQC: a quality control application for high throughput sequence data. Babraham Institute project page, 2016. Available: http://wwwbioinformaticsbabrahamacuk/projects/fastqc

33 Bray NL, Pimentel H, Melsted P, et al. Near-optimal probabilistic RNA-seq quantification. Nat Biotechnol 2016;34:525-7.

34 Soneson C, Love MI, Robinson MD. Differential analyses for RNASeq: transcript-level estimates improve gene-level inferences. F1000Res 2015;4:1521.

35 Law CW, Chen Y, Shi W, et al. voom: precision weights unlock linear model analysis tools for RNA-seq read counts. Genome Biol 2014;15:R29.

36 Krämer A, Green J, Pollard J, et al. Causal analysis approaches in ingenuity pathway analysis. Bioinformatics 2014;30:523-30.

37 Jurtz V, Paul S, Andreatta M, et al. NetMHCpan-4.0: improved peptide-MHC class I interaction predictions integrating eluted ligand and peptide binding affinity data. $J$ Immunol 2017;199:3360-8.

38 Rammensee H, Bachmann J, Emmerich NP, et al. SYFPEITHI: database for MHC ligands and peptide motifs. Immunogenetics 1999;50:213-9.

39 Benjamini Y, Drai D, Elmer G, et al. Controlling the false discovery rate in behavior genetics research. Behav Brain Res 2001;125:279-84.

40 Maris JM, Hogarty MD, Bagatell R, et al. Neuroblastoma. Lancet 2007:369:2106-20.

41 Park JR, Kreissman SG, London WB, et al. Effect of tandem autologous stem cell transplant vs single transplant on event-free survival in patients with high-risk neuroblastoma: a randomized clinical trial. JAMA 2019;322:746-55.

42 Ladenstein R, Pötschger U, Pearson ADJ, et al. Busulfan and melphalan versus carboplatin, etoposide, and melphalan as highdose chemotherapy for high-risk neuroblastoma (HR-NBL1/SIOPEN): an international, randomised, multi-arm, open-label, phase 3 trial. Lancet Oncol 2017;18:500-14.

43 Yarchoan M, Johnson BA, Lutz ER, et al. Targeting neoantigens to augment antitumour immunity. Nat Rev Cancer 2017;17:209-22.

44 Pugh TJ, Morozova O, Attiyeh EF, et al. The genetic landscape of high-risk neuroblastoma. Nat Genet 2013;45:279-84.

45 Voron T, Colussi O, Marcheteau E, et al. VEGF-A modulates expression of inhibitory checkpoints on CD8+ T cells in tumors. $J$ Exp Med 2015;212:139-48.

46 Sade-Feldman M, Jiao YJ, Chen JH, et al. Resistance to checkpoint blockade therapy through inactivation of antigen presentation. Nat Commun 2017:8:1136

47 Sahin U, Derhovanessian E, Miller M, et al. Personalized RNA mutanome vaccines mobilize poly-specific therapeutic immunity against cancer. Nature 2017;547:222-6.

48 Zaretsky JM, Garcia-Diaz A, Shin DS, et al. Mutations associated with acquired resistance to PD-1 blockade in melanoma. N Engl $\mathrm{J}$ Med 2016;375:819-29.

49 Amankulor NM, Kim Y, Arora S, et al. Mutant IDH1 regulates the tumorassociated immune system in gliomas. Genes Dev 2017;31:774-86.

50 Coelho MA, de Carné Trécesson S, Rana S, et al. Oncogenic RAS signaling promotes tumor immunoresistance by stabilizing PD-L1 mRNA. Immunity 2017;47:e1086:1083-99.

51 Miao D, Margolis CA, Vokes NI, et al. Genomic correlates of response to immune checkpoint blockade in microsatellite-stable solid tumors. Nat Genet 2018;50:1271-81.

52 Cristescu R, Mogg R, Ayers M, et al. Pan-tumor genomic biomarkers for PD-1 checkpoint blockade-based immunotherapy. Science 2018;362. doi:10.1126/science.aar3593. [Epub ahead of print: 1210 2018].

53 Asgharzadeh S, Salo JA, Ji L, et al. Clinical significance of tumorassociated inflammatory cells in metastatic neuroblastoma. $\mathrm{J}$ Clin Oncol 2012;30:3525-32. 
54 Steidl C, Lee T, Shah SP, et al. Tumor-Associated macrophages and survival in classic Hodgkin's lymphoma. N Engl J Med 2010;362:875-85.

55 Ueno T, Toi M, Saji H, et al. Significance of macrophage chemoattractant protein-1 in macrophage recruitment, angiogenesis, and survival in human breast cancer. Clin Cancer Res 2000;6:3282-9.

56 Wei JS, Kuznetsov IB, Zhang S, et al. Clinically Relevant Cytotoxic Immune Cell Signatures and Clonal Expansion of T-Cell Receptors in High-Risk MYCN-Not-Amplified Human Neuroblastoma. Clin Cancer Res 2018;24:5673-84.

57 Gentles AJ, Newman AM, Liu CL, et al. The prognostic landscape of genes and infiltrating immune cells across human cancers. Nat Med 2015;21:938-45.

58 Kasim M, Heß V, Scholz H, et al. Achaete-Scute homolog 1 expression controls cellular differentiation of neuroblastoma. Front Mol Neurosci 2016;9:156.

59 Wang L, Tan TK, Durbin AD, et al. ASCL1 is a MYCN- and LMO1dependent member of the adrenergic neuroblastoma core regulatory circuitry. Nat Commun 2019;10:5622.
60 Augustyn A, Borromeo M, Wang T, et al. ASCL1 is a lineage oncogene providing therapeutic targets for high-grade neuroendocrine lung cancers. Proc Natl Acad Sci U S A 2014:111:14788-93.

61 Rheinbay E, Suvà ML, Gillespie SM, et al. An aberrant transcription factor network essential for Wnt signaling and stem cell maintenance in glioblastoma. Cell Rep 2013;3:1567-79.

62 Jankowski MP, Cornuet PK, Mcllwrath S, et al. SRY-box containing gene 11 (Sox11) transcription factor is required for neuron survival and neurite growth. Neuroscience 2006;143:501-14.

63 Decaesteker B, Brouwer SD, Vloed FD. Abstract 5506: Sox11 acts as part of the MYCN-WEE1 regulatory protein complex implicated in neuroblastoma. Cancer Research 2017;77:5506.

64 Lu C, Paschall AV, Shi H, et al. The MLL1-H3K4me3 Axis-Mediated PD-L1 expression and pancreatic cancer immune evasion. J Nat/ Cancer Inst 2017;109. doi:10.1093/jnci/djw283. [Epub ahead of print: 2801 2017].

65 Horn LA, Fousek K, Palena C. Tumor plasticity and resistance to immunotherapy. Trends Cancer 2020;6:432-41. 